\title{
Prioritizing Community Partners and Community HIV Workers in the COVID-19 Pandemic
}

\author{
Don Operario $^{1} \cdot$ Elizabeth J. King $^{2} \cdot$ Kristi E. Gamarel $^{2}$
}

Published online: 30 April 2020

(c) Springer Science+Business Media, LLC, part of Springer Nature 2020

There is accumulating evidence documenting inequities in the burden and impact of the COVID-19 pandemic. In the US, COVID-19 has disproportionately affected people of color and communities with pre-existing health disparities [1]. Globally, the COVID-19 pandemic will also continue to impact those most impacted by HIV [2, 3]. Thus, HIV researchers have been prompt to consider the deleterious effects of COVID-19 on populations already burdened by the HIV pandemic [4-6]. Others have drawn attention to lessons from HIV research that can apply to a rigorous COVID-19 response, including the importance of dismantling stigma [7], building multidisciplinary research teams to address health inequalities [8], and sustaining our investment in public health systems [9]. We add to this dialogue by urging the field to bring visibility to community partners who have continually been on the front-line of HIV prevention, education, advocacy, and social care and who now may face exacerbated vulnerabilities in their professional and personal lives. Their public health contributions and concomitant needs must not be ignored.

We use the term community partner to reflect the breadth of individuals and collaborating organizations (typically these are not-for-profit, non-governmental organizations) that HIV researchers and health departments rely on for realworld impact. These partners have been crucial throughout the unfolding history of HIV prevention and treatment programming [10]. This essential workforce is now on the frontline of a dual pandemic, and many might be at personal risk for COVID-19 as they mobilize to compile and disseminate

Don Operario

don_operario@brown.edu

1 Department of Behavioral and Social Sciences, Brown University School of Public Health, 121 South Main Street, Providence, RI 02912, USA

2 Department of Health Behavior and Health Education, School of Public Health, University of Michigan, Ann Arbor, MI, USA resources and basic supplies to their communities [11, 12]. At times their work has been overlooked, taken for granted, or forgotten by the research community during the course of the HIV epidemic [13]. We urge researchers to not overlook their contributions and challenges during the unfolding COVID-19 pandemic and the reorganization of HIV science as we adapt to continuing our research during unprecedented circumstances.

HIV researchers have a responsibility to acknowledge the challenges and develop interventions to support the health and well-being of our community partners. We highlight specific domains that pose unique challenge to these colleagues.

First, community partners may confront elevated risk for exposure to COVID-19. Due to the sociodemographic, geographic, and environmental intersections where HIV and COVID-19 meet, our community partners are likely to have contact with symptomatic and asymptomatic people in the course of delivering HIV prevention, testing, referrals, and social care. HIV-focused community-based organizations must institute policies to acknowledge and mitigate employees' risk for COVID-19 infection. Organizations must ensure provision of personal protective equipment (PPE), COVID19 safety education, and enhanced workplace safety protocols to community partners and project staff who implement service programs and research. Collaborating HIV researchers must help to ensure that these needs are met for their community partners.

In addition, community partners are vulnerable to the economic impacts of COVID-19. Anecdotal stories abound regarding industries shutting down, funding cuts to community-based organizations, employees being furloughed, and populations suffering financial despair. Community partners may similarly experience economic shocks from COVID-19, both at the organizational and personal levels. Financial challenges to HIV organizations and community workers may continue after the "first wave" of COVID-19, especially if community/public HIV program budgets shrink 
or if research priorities migrate away from community-based activities. Indeed, technology-mediated procedures (e.g., video counseling, online surveys) can allow some research protocols to continue in an era of social distancing $[14,15]$. HIV researchers must be cautious to not dispose of their community partners in the move toward mHealth services delivery. To maximize social relevance and impact, HIV prevention researchers must continue collaborations with community partners in the development and delivery of remote research procedures. This not only ensures the sustainable involvement of community partners, but will also ensure that the rollout of these adapted protocols will be ethical and beneficial to the communities served.

Community partners are also vulnerable for both acute and sustained psychosocial consequences of COVID-19. Emotional distress, depression, exhaustion, trauma, and grieving are among the psychosocial responses that community-based service providers may experience. They have also had to adapt their own work to adhere to social distancing guidelines and government orders. HIV researchers must recognize and prioritize efforts to address the psychosocial consequences of front-line human services professionals who work at the community front line.

The professional responsibilities of our community partners are likely to evolve in the aftermath of COVID-19. There have already been indications that community health workers, including those providing community-based HIV services, are strategically poised to implement populationbased measures that educate, screen, and provide referrals to people at risk for COVID-19 [16]. These expanded responsibilities require investments in training, supervision, and resources (e.g., PPE, psychological support) to protect front-line community workers. Additional institutional and structural considerations include workers' burnout, overload, and compensation commensurate to their responsibilities and risk.

At this relatively early stage in the COVID-19 pandemic, HIV researchers have an opportunity to set an agenda that prioritizes the contributions, risks, and needs of our community partners who are at the forefront of public health prevention, testing, and advocacy efforts. We must resist historic tendencies to render their personal risks invisible and their labor disposable $[13,17]$. We have an ethical imperative to respond through explicit acknowledgment, concrete investment, and primary research into the health and economic risks, psychosocial challenges, and professional skills training needed to support our community partners.

Acknowledgements The authors thank the community partners and HIV community workers who have remained on the front-line of the COVID-19 pandemic. The authors are grateful to the Trans Sistas of Color Project-Detroit for their inspiration through demonstrating continued advocacy and care to transgender women of color in Detroit, Michigan.

\section{Compliance with Ethical Standards}

Conflict of interest Each of the authors declare that they have no conflicts of interest.

\section{References}

1. van Dorn A, Cooney RE, Sabin ML. COVID-19 exacerbating inequalities in the US. Lancet. 2020;395(10232):1243-4.

2. Ahmed F, Ahmed N, Pissarides C, Striglitz J. Why inequality could spread COVID-19. Lancet Public Health. 2020. https:// doi.org/10.1016/S2468-2667(20)30085-2.

3. Nkengasong JN, Mankoula W. Looming threat of COVID19 infection in Africa: act collectively, and fast. Lancet. 2020;395(10227):841-2.

4. Carrico AW, Horvath KJ, Grov C, Moskowitz JT, Pahwa S, Pallikkuth S, et al. Double jeopardy: methamphetamine use and HIV as risk factors for COVID-19. AIDS Behav. 2020;7:1.

5. Davey DJ, Bekker L-G, Coates TJ, Myer L. Contracting HIV or contracting SAR-CoV-2 (COVID-19) in pregnancy? Balancing the risks and benefits. AIDS Behav. 2020;13:1.

6. Shiau S, Krause KD, Valera P, Swaminathan S, Halkitis PN. The burden of COVID-19 in people living with HIV: A syndemic perspective. AIDS Behav. 2020;18:1.

7. Logie CH, Turan JM. How do we balance tensions between COVID-19 public health responses and stigma mitigation? Learning from HIV research. AIDS Behav. 2020;13:1-4.

8. Hargreaves J, Davey C, Auerbach J, Blanchard J, Bond V, Bonell $\mathrm{C}$, et al. Three lessons for the COVID-19 response from pandemic HIV. Lancet HIV. 2020;9:1.

9. Validserri RO, Holtgrave DR. Responding to pandemics: what we've learned from HIV/AIDS. AIDS Behav. 2020;9:1.

10. Rhodes SD, Tanner AE, Mann-Jackson L, Alonzo J, Horridge $\mathrm{DN}$, Van Dam CN, et al. Community-engaged research as an approach to expedite advances in HIV prevention, care, and treatment: a call to action. AIDS Educ Prev. 2018;30(3):243-53.

11. Vasquez T. 'We keep us safe': black trans women on the frontlines of the pandemic. In Daily Kos. 2020; https://www.daily kos.com/stories/2020/4/17/1938139/--We-keep-us-safe-Black -trans-women-on-the-frontlines-of-the-pandemic

12. Knoppow ES. Trans Sistas of color project distributes care packages to those in need of COVID-19. In PrideSource. 2020; https ://pridesource.com/article/trans-sistas-of-color-project-distr ibutes-care-packages-to-those-in-need-because-of-covid-19/

13. Parker R. Grassroots activism, civil society mobilization, and the politics of the global HIV/AIDS epidemic. Brown J World Affairs. 2011;17(2):21-37.

14. Saberi P. Research in the time of coronavirus: continuing ongoing studies in the midst of the COVID-19 pandemic. AIDS Behav. 2020;18:1-4.

15. Hightow-Weidman LB, Muessig K, Claude K, Roberts J, Zlotorzynksa M, Sanchez T. Maximizing digital interventions for youth in the midst of Covid-19: lessons from the Adolescent Trials Network for HIV Interventions. AIDS Behav. 2020;18:1.

16. Hoff T, Kates J, Dawson L, Kistler R. Managing HIV during COVID-19: working to end one epidemic while confronting another. In KKF. 2020; https://www.kff.org/coronavirus-polic $\mathrm{y}$-watch/managing-hiv-during-covid-19-working-to-end-oneepidemic-while-confronting-another/ 
17. African American Policy Forum. Under the blacklight: the intersectional failures that COVID lays bare (part 2). Moderated by Crenshaw K; April 2020. https://aapf.org/under-the-blacklight -covid19
Publisher's Note Springer Nature remains neutral with regard to jurisdictional claims in published maps and institutional affiliations. 